\title{
HERMITE INTERPOLATION AND INEQUALITIES INVOLVING WEIGHTED AVERAGES OF $n$-CONVEX FUNCTIONS
}

\author{
JOSIP PEČARIĆ AND MARJAN PRALJAK
}

Abstract. By using Hermite interpolation we obtain Popoviciu-type inequalities containing sums $\sum_{i=1}^{m} p_{i} f\left(x_{i}\right)$, where $f$ is an $n$-convex function. We also give integral analogues of the results, as well as bounds for integral remainders of identities associated with the obtained inequalities.

Mathematics subject classification (2010): 26D15, 26D10.

Keywords and phrases: $n$-convex functions, Hermite interpolation, Čebyšev functional.

\section{REFERENCES}

[1] M. Adil Khan, N. Latif, J. PeČArić, Generalization of majorization theorem, J. Math. Inequal. 9 (2015), 847-872

[2] R. P. Agarwal, P. J. Y. Wong, Error Inequalities in Polynomial Interpolation and Their Applications, Kluwer Academic Publishers, Dordrecht/Boston/London, 1993.

[3] S. I. BUtT, K. A. KhAN, J. PeČARIĆ, Popoviciu type inequalities via Green function and generalized Montgomery identity, Math. Ineq. Appl. 18 (2015), 1519-1538.

[4] P. Cerone AND S. S. DRAGOMiR, Some new Owstrowski-type bounds for the Čebyšev functional and applications, J. Math. Inequal., 8 (1) (2014), 159-170.

[5] J. PEČARIĆ, On Jessen's Inequality for Convex Functions, III, J. Math. Anal. Appl., 156 (1991), 231239.

[6] J. E. PeČarić, F. Proschan And Y. L. Tong, Convex functions, partial orderings and statistical applications, Academic Press, New York, 1992.

[7] T. Popoviciu, Notes sur les fonctions convexes d'orde supérieur III, Mathematica (Cluj) 16, (1940), $74-86$.

[8] T. Popoviciu, Notes sur les fonctions convexes d'orde supérieur IV, Disqusitiones Math. 1, (1940), $163-171$.

[9] T. Popoviciu, Notes sur les fonctions convexes d'orde supérieur IX, Bull. Math. Soc. Roumaine Sci. 43, (1941), 85-141.

[10] T. Popoviciu, Les fonctions convexes, Herman and Cie, Editeurs, Paris 1944. 